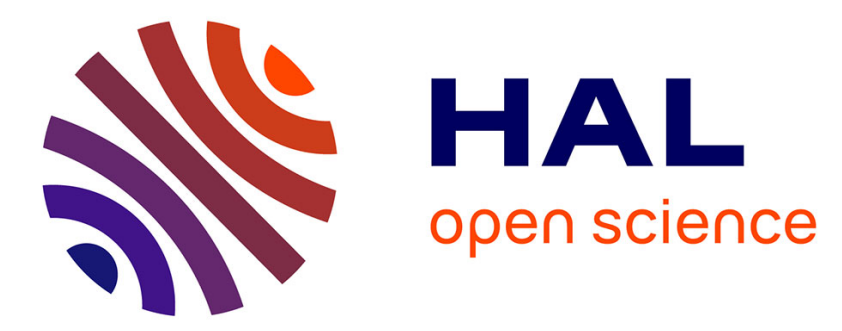

\title{
RANS Simulations of Supercavity Flows
}

\author{
Christian Pellone, Thierry Maître, Jean-Pierre Franc
}

\section{To cite this version:}

Christian Pellone, Thierry Maître, Jean-Pierre Franc. RANS Simulations of Supercavity Flows. Journal of Ship Research, 2010, 54 (3), pp.161-173. hal-00936649

\section{HAL Id: hal-00936649 \\ https://hal.science/hal-00936649}

Submitted on 5 May 2020

HAL is a multi-disciplinary open access archive for the deposit and dissemination of scientific research documents, whether they are published or not. The documents may come from teaching and research institutions in France or abroad, or from public or private research centers.
L'archive ouverte pluridisciplinaire HAL, est destinée au dépôt et à la diffusion de documents scientifiques de niveau recherche, publiés ou non, émanant des établissements d'enseignement et de recherche français ou étrangers, des laboratoires publics ou privés. 


\title{
RANS Simulations of Supercavity Flows
}

\author{
C. Pellone, ${ }^{*}$ T. Maître, ${ }^{\dagger}$ and J. P. Franc* \\ ${ }^{*}$ Research Scientist, CNRS, Centre National de la Recherche Scientifique, Grenoble, France \\ ${ }^{\dagger}$ Research Scientist, INPG, Institut National Polytechnique de Grenoble, Grenoble, France
}

\begin{abstract}
This work aims at evaluating the capacity and limitations of conventional Reynoldsaveraged Navier-Stokes (RANS) techniques to numerically simulate supercavity flows. The configuration is that of a two-dimensional (2D) symmetrical supercavitating wedge investigated experimentally by Michel (1974). Mesh effect is studied in detail under noncavitating conditions. The computational grid is refined in the region where cavitation develops in order to accurately track the supercavity. The effect of tunnel height is also analyzed, and the height finally chosen was large enough to simulate an infinite flow-field. The cavitating flow is treated as a homogeneous mixture of variable density. To account for vaporization and condensation, an additional continuity equation for the vapor (or the liquid) is solved with an appropriate source term expressing mass transfer between the two phases. The effect of nuclei concentration on the vaporization rate and then on the development of the supercavity is investigated. Results obtained using two different RANS codes are compared. They are also compared with experimental data and with inviscid solutions including a nonlinear boundary element method. They concern in particular cavity length and shape, pressure distribution, and drag coefficient. Under unsteady conditions, a special attention is paid to the characteristic growth time of the supercavity following a sudden pressure drop, from noncavitating conditions.
\end{abstract}

Keywords: cavitation; hydrodynamics (general)

\section{Introduction}

SuPERCAVITATION is a common feature of high-speed hydrodynamics. When a submerged body moves at high speed (from typically several tens of $\mathrm{m} / \mathrm{s}$ up to the speed of sound in water and even beyond), a cavitation bubble is formed from its nose. The term supercavitation refers to the case when the whole body is enveloped by the bubble, which then closes inside the liquid, far enough from any wall. A positive outcome of supercavitation is the drastic reduction of skin friction and drag compared with the fully wetted case (Kirschner et al. 2001, Savchenko 2001). A supercavity can be generated either by spontaneous vaporization of the liquid or by supplying gas and then creating a ventilated cavity whose behavior is similar to that of a natural cavity, although some differences exist because of the noncondensable nature of the injected gas. Supercavitation is also observed during water entries (Shi \& Kume 2004) or on surface-piercing propellers (Kinnas 2001).

In the past, various techniques have been developed to model supercavitating flows as analytic methods based on the hodograph technique (Birkhoff \& Zarantonello 1957), linearized theories (Tulin 1964), analytic methods based on the slender body approximation, and the Logvinovich independence principle of cavity expansion (Serebryakov 2002). The boundary element method has been also widely used for the numerical computation of supercavity flows (Pellone \& Rowe 1981). In all cases, the modeling of the closure region of the cavity is a major problem because of local unsteadiness, turbulence, and entrainment mechanisms, and various cavity closure models were developed to overcome these difficulties (see e.g., Franc \& Michel 2004).

CFD tools are currently widely used to predict the development of cavitation in liquid flows. The most critical problem for the simulation of cavitating flows, unlike that for single-phase flows, is probably the choice of the cavitation model together with the turbulence model. 
A commonly used approach for modeling turbulent cavitating flows is based on the Reynolds-averaged Navier-Stokes (RANS) equations. For RANS techniques, each variable is divided into a mean and a fluctuating component, and the RANS equations are closed using a turbulence model to account for Reynolds stresses. Two-equation models are often used, as the $k$ - $\varepsilon$ model. As such turbulence models were originally developed for single-phase flows, they are generally not fully satisfactory for cavitating flows where large density variations are encountered between the liquid and its vapor. A typical limitation of classic single-phase turbulence models when applied to cavitating flows is their relative failure to correctly predict unsteady cavitation as cloud shedding. To allow the development of unsteadiness within cavitating flows, which are often inhibited by classic turbulence models, empirical corrections have been developed, as an artificial reduction of the eddy viscosity in two-phase regions (see e.g., CoutierDelgosha et al. 2005, Dular et al. 2005).

RANS simulations of cavitating flows are most generally based on the homogeneous two-phase flow approach. The liquid/vapor mixture is considered a pseudo fluid of variable density, whose value strongly depends on the void fraction. To account for phase change, a cavitation model has to be implemented. Different variants have been proposed.

A first class of models specifies a constitutive law for this pseudo fluid. As an example, the barotropic model assumes a simple relationship between pressure and density with a more or less sudden drop of density from liquid to vapor when the pressure crosses the vapor pressure (Pouffary et al. 2003, Rapposelli \& d'Agostino 2003). The pioneering approach developed by Kubota et al. (1992) assumes that the liquid contains cavitation nuclei that grow in low-pressure regions. Their radius and then the void fraction are computed by solving a modified Rayleigh-Plesset equation for the bubble cluster including interactions between bubbles. The density of the liquid/vapor mixture can then be computed, and the system of partial differential equations for the pseudo fluid can be solved.

Another class of models solves an additional continuity equation for the vapor phase with a source term modeling vaporization and condensation through the interface. Several equations have been proposed in the literature to compute the vaporization rate (Merkle et al. 1998, Kunz et al. 1999, Lindau et al. 2002, Singhal et al. 2002, Vortmann et al. 2003, Yuan \& Schnerr 2003). Some of them have been compared by Senocak and Shyy (2002) and Frikha et al. (2008). It generally turns out that most models give comparable overall results even though a few differences are observed, particularly at cavity closure. Several cavitation models assume that the liquid carries microbubbles. The vaporization rate is then deduced from the bubble number density and their growing rate generally inferred from a simplified version of Rayleigh equation. This is the approach selected in the present work.

Let us also mention techniques that are not based on the mixture model assumption but assume that liquid and vapor are separated by a sharp interface without any possibility of interpenetration. In these techniques (see in particular Diéval 1999, Diéval et al. 2000), only the liquid flow is computed by solving the NavierStokes equations, whereas the interface (on which the pressure is set to the vapor pressure) is reconstructed and propagated at each time step using the standard volume of fluid (VOF) technique.

More recently, large eddy simulation (LES) techniques or detached eddy simulation (DES) techniques have been developed and compared with RANS simulations. LES and DES techniques are known to require considerably larger computational times but resolve turbulence vortex shedding of separated flows better than do RANS techniques (Spalart 2009, Breuer et al. 2003). LES was applied to the computation of cavitating flows in particular by Persson et al. (2006), Wang and Ostoja-Starzewski (2007), and Huuva (2008). These three-dimensional simulations led to much more realistic predictions of the unsteady behavior of partial cavities, especially concerning the three-dimensional and unsteady features of cavitating vortex shedding in the closure region of the cavity, which is definitely the most critical region.

RANS techniques are commonly used to simulate various cavitating configurations as partial cavitation on hydrofoils (Saito et al. 2003, Wu et al. 2003) or cavitating flows in hydraulic machinery (e.g., Coutier-Delgosha et al. 2005). In such configurations where cavitation is generally not very much developed, the cavitating areas have a maximum void fraction usually smaller than unity, and the cavity is actually a mixture of liquid and vapor. The situation is quite different in the case of supercavitation, where a well-defined interface separates the liquid flow from the full vapor cavity along most of the supercavity, except in its closure region. A key issue is then to know whether conventional RANS techniques are appropriate or not to the simulation of supercavitation. This is the main goal of the present work.

In the present work, the performances of two RANS techniques with respect to the simulation of supercavity flows are evaluated. One is the commercial code Fluent (version 6.0.12) and the other is the code Cavka developed at the University of München by G.H. Schnerr (Schnerr \& Sauer 2001, Yuan \& Schnerr 2003). Both are based on a homogeneous approach with a $k$ - $\omega$ turbulence model of Wilcox (Sauer \& Schnerr 2000) and use similar cavitation models assuming a vaporization rate proportional to the square root of the difference between the vapor pressure and the local pressure. The test case is that of a supercavity flow in

\section{Nomenclature}

\footnotetext{
$c=$ Chord of the foil (m)

$C_{\mathrm{D}}=$ Drag force coefficient

$C_{\mathrm{Dp}}=$ Pressure drag force coefficient

$C_{\mathrm{p}}=$ Pressure coefficient

$H=$ Tunnel height $(\mathrm{m})$

$l_{\mathrm{c}}=$ Cavity length $(\mathrm{m})$

$M_{\mathrm{v}}=$ Vapor mass (mg)

$N=$ Nuclei concentration per unit volume (nuclei $/ \mathrm{m}^{3}$ )

$p=$ Static pressure $(\mathrm{Pa})$
}

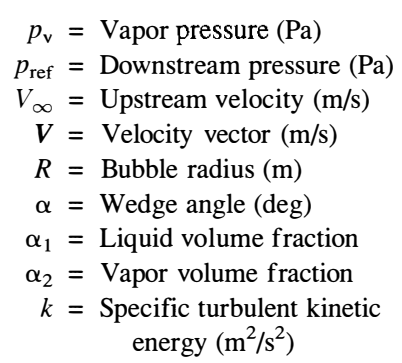

$\mu=$ Mixture molecular viscosity $[\mathrm{kg} /(\mathrm{m} \cdot \mathrm{s})]$

$\mu_{1}=$ Liquid molecular viscosity $[\mathrm{kg} /(\mathrm{m} \cdot \mathrm{s})]$

$\mu_{2}=$ Vapor molecular viscosity $[\mathrm{kg} /(\mathrm{m} \cdot \mathrm{s})]$

$\omega=$ Specific turbulent energy dissipation rate $\left[\mathrm{m}^{2} / \mathrm{s}^{3}\right]$

$\rho=$ Mixture density $\left(\mathrm{kg} / \mathrm{m}^{3}\right)$

$\rho_{1}=$ Liquid density $\left(\mathrm{kg} / \mathrm{m}^{3}\right)$

$\rho_{2}=$ Vapor density $\left(\mathrm{kg} / \mathrm{m}^{3}\right)$

$\sigma=$ Cavitation number 
the wake of a two-dimensional (2D) wedge as investigated experimentally by Michel (1974). In the steady case, both RANS techniques are compared with experimental data, a boundary element method, and an analytic computation. The unsteady response of the flow to a sudden drop in pressure from noncavitating conditions to a largely developed supercavity is also investigated using both codes.

\section{Numerical study}

The numerical model follows the homogeneous two-phase flow approach and resolves cavitation by applying a bubble growth model. To model turbulence the effective dynamic viscosity $\mu_{\text {eff }}=\mu+\mu_{t}$ is used instead of the classic molecular viscosity $\mu$. The turbulent viscosity $\mu_{t}$ is computed using the Wilcox model (Wilcox 1998).

\subsection{Governing equations}

The continuity equations for each phase of the cavitating flow are:

$$
\frac{\partial\left(\alpha_{i} \rho_{i}\right)}{\partial t}+\operatorname{div}\left(\alpha_{i} \rho_{i} \boldsymbol{V}_{i}\right)=\Gamma_{i} \quad i \in[1,2]
$$

where $t$ stands for time, $\boldsymbol{V}$ for the velocity, $\rho$ for the density, $\alpha$ for the volume fraction, and $\Gamma$ for the mass exchange rate per unit volume at the interface. Subscript $i=1$ indicates the liquid phase and $i=2$ the vapor phase.

The two-phase mixture is considered as a homogeneous pseudo fluid with the assumption of no slip between the phases; that is, $V_{1}=V_{2}=\boldsymbol{V}$. The pseudo density $\rho$ and the pseudo dynamic viscosity $\mu$ are defined as:

$$
\begin{gathered}
\rho=\alpha_{1} \rho_{1}+\alpha_{2} \rho_{2} \\
\mu=\alpha_{1} \mu_{1}+\alpha_{2} \mu_{2}
\end{gathered}
$$

$\rho_{\mathrm{i}}$ and $\mu_{\mathrm{i}}$ are, respectively, the density and the dynamic viscosity of each $i$ phase and are supposed to be constant.

Introducing the particle derivative $D / D t=\partial / \partial t+V \cdot \operatorname{grad}$ and assuming the equality of the mass rates per unit volume $\left(\Gamma=\Gamma_{2}=\right.$ $-\Gamma_{1}$ ) exchanged between the liquid and the vapor phases, a simple combination of the two continuity equations (1) provides the continuity equation for the mixture:

$$
\frac{D \rho}{D t}+\rho \operatorname{div}(\boldsymbol{V})=0
$$

with the compatibility condition:

$$
\alpha_{1}+\alpha_{2}=1
$$

Schnerr and Sauer (2001) notice that the nonconservative form of the continuity equation (4) limits the numerical difficulties because of the strong variation of density between liquid and vapor. In addition, this form presents the advantage that the volume fluxes are continuous at the cell interfaces.

By extracting $\operatorname{div}(\boldsymbol{V})$ from equation (4) and using equations (2) and (5), the two equations (1) can be rewritten in the form of transport equations for the two volume fractions $\alpha_{1}$ and $\alpha_{2}$ :

$$
\partial\left(\alpha_{1}\right) \cdot \operatorname{div}\left(\alpha_{1} \boldsymbol{V}\right)=-\frac{\Gamma}{\rho_{1}}=\frac{\rho_{2}}{\rho} \frac{D \alpha_{1}}{D t}
$$

$$
\frac{\partial\left(\alpha_{2}\right)}{\partial t}+\operatorname{div}\left(\alpha_{2} \boldsymbol{V}\right)=+\frac{\Gamma}{\rho_{2}}=\frac{\rho_{1}}{\rho} \frac{D \alpha_{2}}{D t}
$$

Introducing the static pressure $p$ and neglecting gravity, the momentum equation for the mixture writes:

$$
\frac{\partial(\rho \boldsymbol{V})}{\partial t}+\operatorname{Div}\left[\rho \boldsymbol{V} \otimes \boldsymbol{V}+\left(p+\frac{2}{3} \mu \operatorname{div}(\boldsymbol{V})\right) \overline{\bar{G}}\right]=\operatorname{Div}(2 \mu \stackrel{\overline{\boldsymbol{D}}}{)}
$$

where $\otimes$ designates the tensorial product of two vectors, Div is the vectorial divergence operator, $\bar{G}$ is the metric tensor, and $\bar{D}$ is the rate of strain tensor.

The system of equations to be solved consists of the compatibility relation (5), the two transport equations for each phase (6) and (7) [or one of these and the continuity equation for the mixture (4)], and the vectorial momentum equation (8). The unknown variables are $\alpha_{1}, \alpha_{2}, \Gamma, p$, and $\boldsymbol{V}$. In order to close the system of equations, an additional equation is needed. It is obtained using an appropriate cavitation model.

\subsection{Cavitation model}

The vaporization or condensation process is modeled here by seeding the flow field with microbubbles and computing their evolution on the basis of the Rayleigh-Plesset equation (Franc \& Michel 2004) as proposed originally by Kubota et al. (1992) and generalized by Singhal et al. (2002). Assuming that all spherical bubbles have the same radius $R$ and then the same volume $\vartheta=$ $4 / 3 \pi R^{3}$, denoting by $N$ the vapor nuclei concentration per unit volume of pure liquid (so $\alpha_{2}=9 N \alpha_{1}$ ) and using the compatibility condition (5), the two volume fractions $\alpha_{1}$ and $\alpha_{2}$ and their particle derivatives can be expressed as:

$$
\left\{\begin{array}{l}
\alpha_{2}=1-\alpha_{1}=\frac{\vartheta N}{1+\vartheta N} \\
\frac{D \alpha_{2}}{D t}=-\frac{D \alpha_{1}}{D t}=\frac{N \dot{\vartheta}}{(1+\vartheta N)^{2}}
\end{array}\right.
$$

where $\dot{\vartheta}=4 \pi R^{2} \dot{R}$.

Notice that, for a fixed value of $N$, when $\alpha_{1}=0$ (pure vapor), $\vartheta$ becomes infinite, and when $\alpha_{1}=1$ (pure liquid), $\vartheta$ becomes zero. In order to model both the bubble growth and collapse processes, the Rayleigh equation is used. Assuming that the bubble pressure is equal to the equilibrium vapor pressure $p_{\mathrm{v}}$, the interface velocity is directly related to the pressure difference $p-p_{\mathrm{v}}$ by:

$$
\dot{R}=\varepsilon \sqrt{\frac{2}{3}} \frac{\left|p_{\mathrm{v}}-p\right|}{\rho_{1}}
$$

$\varepsilon$ designates the sign of $\left(p_{\mathrm{v}}-p\right)$, so that the bubble grows when $p<p_{\mathrm{v}}$ and collapses when $p>p_{\mathrm{v}}$, the growth rate being zero if $p=p_{\mathrm{v}}$. The growth rate given by equation (10) is actually the asymptotic growth rate deduced from Rayleigh equation when assuming the interface acceleration $\ddot{R}$ is negligible.

As a matter of fact, the present cavitation model disregards the dynamic term of the Rayleigh equation. This is the case for most cavitation models that generally evaluate the mass transfer between the two phases by the sum of a vaporization and a condensation rate. Both are computed usually from the local void fraction and the local pressure difference relatively to the vapor 
pressure (Frikha et al. 2008) and therefore do not include any dynamic term contrary to the original model of Kubota et al. (1992), which is keeping the acceleration of the bubble interface in its formulation. It is presently difficult to assess the impact of neglecting the dynamic term on the simulation results and particularly on the overall dynamics of the supercavity.

Equations (9) and (10) provide the additional relation that closes the governing equations system:

$$
\Gamma=\frac{\rho_{1} \rho_{2}}{\rho} \begin{gathered}
N 4 \pi R^{2} \\
(1+9 N)^{2}
\end{gathered} \sqrt[3]{\frac{2}{3} \frac{\left|p_{v}-p\right|}{\rho_{1}}}
$$

The use of these relations requires the knowledge of the nuclei concentration $N$, which is not easily measured. Briançon-Marjollet et al. (1990), using a cavitation susceptibility meter, measured about $10^{7}$ nuclei $/ \mathrm{m}^{3}$ in a conventional cavitation tunnel seeded with nuclei. Most computations presented in this paper have been conducted with a nuclei concentration of $10^{8}$ nuclei $/ \mathrm{m}^{3}$. We could observe that a too small nuclei density (typically of the order of $10^{5}$ nuclei $/ \mathrm{m}^{3}$ ) may lead to unrealistic results, whereas an asymptotic behavior is reached for high enough values of the nuclei concentration as shown in section 4.2.

\subsection{Numerical scheme}

The governing equations are integrated on an individual control volume to obtain the discretized equations in both space and time. The numerical formulation uses a cell-centered finite volume method for variables $\boldsymbol{V}, p, k, \omega$, and $\alpha_{1}$ or $\alpha_{2}$.

Assuming that the solution is known at time $t$, an iterative procedure is carried out to obtain the converged solution at time $t+\Delta t$. If the calculation has just begun, the solution is updated based on the initialized solution. The governing equations are solved sequentially, that is, segregated from one another. Each discrete equation is linearized implicitly in space, using a second-order upwind scheme. Concerning the discretization in time, a Euler implicit formulation is used for the continuity, momentum, and turbulence equations. Second-order numerical schemes are used.

The two codes Fluent and Cavka mainly differ by the manner of solving one of the two transport equation (6) or (7). In the case of Fluent, equation (7) relative to the void fraction is selected and implicitly discretized in time. In the case of Cavka, equation (6) relative to the liquid phase is selected and explicitly discretized in time. The mode of resolution between time $t$ and $t+\Delta t$ is detailed below for both approaches. At the current time $t+\Delta t$, the current iteration is denoted by $i+1$, all variables being known at the previous iteration $i$.

\subsection{Solution procedure of Fluent}

At the current iteration $i+1$, the calculation stages are outlined as:

1. The momentum equation (8) is first solved using the $i$ iteration values for pressure and face mass fluxes to update the velocity field.

2. Because this velocity field may not satisfy the continuity equation locally, a Poisson-type equation for pressure is derived from the continuity equation (4) and the linearized momentum equations. This pressure correction equation is then solved to obtain the necessary corrections for pressure, such that continuity is satisfied (SIMPLE algorithm, Ferziger \& Peric 1996).

3. Then, the turbulence equations for $k$ and $\omega$ are solved using current values of the velocity and pressure updated in stages 1 and 2 .

4. The transport equation (7) for the vapor volume fraction $\alpha_{2}$ is solved using the current values of pressure and velocity. Equation (7) is implicitly discretized with time and the source term $\Gamma / \rho_{2}$ [given by equation (11)] is calculated using the previous $i$ iteration values of $\alpha_{1}$ and $\alpha_{2}$.

These stages are continued until the convergence criteria are met.

\subsection{Solution procedure of Cavka}

First, the transportation of the liquid volume fraction $\alpha_{1}$ is computed explicitly by using equation (6) in which, the velocity $\boldsymbol{V}$ at any point and the source term $-\Gamma / \rho_{1}$ [given by equation (11)] are provided by the previous time step $t$. The new volume fractions are thus obtained, as well as the new mixture density $\rho$. Then, the momentum equation (8) is implicitly discretized with subiterations and coupled with the continuity equation (4) via the SIMPLE algorithm, similar to that of Fluent.

In order to capture the interface accurately, the high-level interpolation method CICSAM (Compressive Interface Capturing Scheme for Arbitrary Meshes; Ubbink 1997) is used for equation (6). After integration on a given cell, this equation gives the void fraction at the center of the cell and at the current time step as a function of the values of the void fraction on the faces and the volumetric fluxes through the faces at the previous time step. The required values of the void fraction on faces are estimated by the CICSAM scheme in the framework of the standard upwind/ donor/acceptor approach. The technique makes use of the Normalized Variable Diagram (NVD) (Leonard 1991). The CICSAM scheme combines the Convection Boundedness Criteria (CBC) (Ubbink \& Issa 1999) with the Ultimate Quickest (UQ) differencing scheme (Leonard 1991), which both depend on the Courant number. The normalized face value is computed by combining the values obtained with these two schemes through a weighting factor that depends on the interface orientation and the fluxing direction (Greaves 2004, Waclawczyk \& Koronowicz 2008). The CICSAM scheme is known to prevent artificial smearing of the interface resulting from numerical diffusion (Waclawczyk \& Koronowicz 2005). The method is explained in detail in Sansone (2007) and was used successfully by the author to model cavitation in Darrieus turbines.

\section{Test case}

Computations are carried out on a supercavitating wedge in a cavitation tunnel limited by an upper and a lower wall. The noslip condition is applied on the wedge, whereas a slip condition is applied on the tunnel walls. The overall configuration is twodimensional and symmetrical with respect to axis Ox (Fig. 1).

The wedge has a chord length $c=60.5 \mathrm{~mm}$ and a vertex angle $\alpha=16 \mathrm{deg}$. For most computations, the tunnel height has been chosen equal to $H=940 \mathrm{~mm}$, that is, approximately 15.5 chord lengths. As shown below, this relatively large height ensures that the influence of the tunnel walls is negligible and that the flow field can be considered almost infinite. 


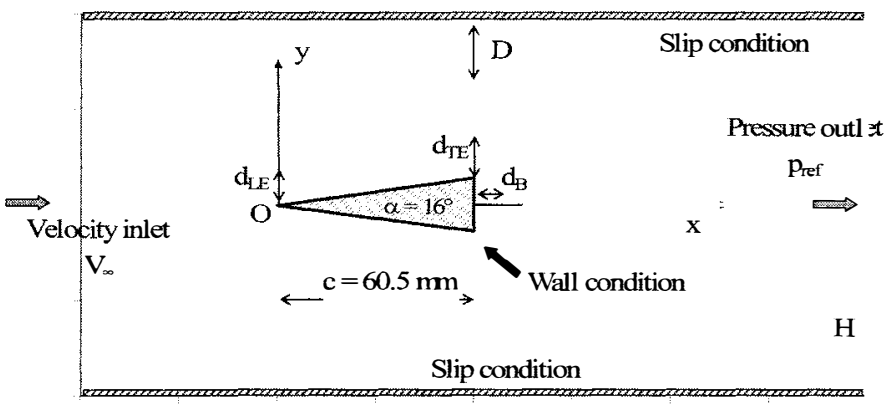

Fig. 1 Schematic view and boundaries conditions

The velocity inlet condition $\left(V_{\infty}\right)$ is applied 3 chord lengths upstream of the leading edge, whereas the pressure outlet condition $\left(p_{\text {ref }}\right)$ is applied 9 chord lengths downstream of the wedge base, so that the whole computational domain has a length equal to 13 chord lengths.

The upstream velocity is assumed to be uniform and equal to $V_{\infty}=10 \mathrm{~m} / \mathrm{s}$. The liquid is water at room temperature with density $\rho_{1}=998 \mathrm{~kg} / \mathrm{m}^{3}$, whereas the density of vapor is $\rho_{2}=0.017 \mathrm{~kg} / \mathrm{m}^{3}$. The density ratio is then about $1 / 60,000$. The molecular viscosities of liquid and vapor, although negligible with respect to turbulent viscosity, are, respectively, $\mu_{1}=1.010^{-3} \mathrm{~kg} / \mathrm{m} \cdot \mathrm{s}$ and $\mu_{2}=8.810^{-3} \mathrm{~kg} / \mathrm{m} \cdot \mathrm{s}$. Reynolds number based on chord length is about $6 \times 10^{5}$.

The cavitation number is defined as usually by:

$$
\sigma=\frac{p_{\text {ref }}-p_{\mathrm{v}}}{1 / 2 p_{1} V_{\infty}^{2}}
$$

Vapor pressure is $p_{\mathrm{v}}=2329 \mathrm{~Pa}$, whereas the reference pressure $p_{\text {ref }}$ is chosen as the downstream pressure. Pressure coefficient and drag coefficient are defined by:

$$
\begin{gathered}
C_{\mathrm{p}}=\frac{p-p_{\mathrm{ref}}}{1 / 2 \rho_{1} V_{\infty}^{2}} \\
C_{\mathrm{D}}=\frac{F_{\mathrm{x}}}{1 / 2 \rho_{1} c V_{\infty}^{2}}
\end{gathered}
$$

where $F_{\mathrm{x}}$ is the component along the horizontal axis Ox of the fluid force on the wedge per unit span length.

At inlet, the parameters of the $k$ - $\omega$ turbulence model are $2 \%$ for the turbulence level and about the wedge base thickness for the characteristic length scale. Wall laws are used for turbulent boundary layer conditions on the wedge.

\subsection{Influence of the mesh}

Before conducting a comparative analysis of both simulations for cavity flows, a preliminary investigation is carried out to examine the influence of the mesh. This investigation is conducted for fully wetted flow, under steady conditions, using Fluent.

The mesh is structured and satisfies the following conditions. On the upper and lower sides of the wedge, the ratio between cell thicknesses at leading edge and trailing edge is taken as $d_{\mathrm{LE}} / d_{\mathrm{TE}}=$ 0.1 . This value corresponds approximately to the ratio of the boundary layer thicknesses at leading edge and trailing edge for the corresponding Reynolds number. The turbulent boundary layer thickness at trailing edge is about $1.6 \mathrm{~mm}$.
The length $d_{\mathrm{B}}$ of the first cell behind the obstacle satisfies $d_{\mathrm{B}} / d_{\mathrm{TE}}=1.5$. Furthermore, since the ratio $H / c$ is relatively large, the flow can be considered almost uniform on the tunnel walls and the thickness $D$ of the first cell along the walls can be large. In practice, we choose $D / d_{\mathrm{TE}} \approx 400$. In both directions, the ratio between the size of two adjacent cells never exceeds 1.2.

Six different meshes have been used to investigate the influence of the computational mesh. The number of cells is, respectively, 3,184, 7,280, 15,904, 18,120, 51,840, and 76,176 and corresponds to the six following values of $d_{\mathrm{TE}}: 1.1,0.68,0.40$, $0.32,0.20$, and $0.10 \mathrm{~mm}$. Figure 2 shows a typical computational mesh of 18,120 cells obtained for $d_{\mathrm{TE}}=0.32 \mathrm{~mm}$. Let us observe that the mesh is refined in the wake of the wedge where the supercavity is expected.

Figure $3 a$ shows that there is almost no influence of the mesh on the pressure distribution on the wedge. The difference never exceeds $1 \%$. As for the drag coefficient, Fig. $3 b$ shows that, for $d_{\mathrm{TE}} \leq 0.32 \mathrm{~mm}$; that is, for a number of cells greater than 18,120 , the drag coefficient is stable within less than $1 \%$. As a conclusion of this preliminary investigation, the mesh with 18,120 cells is chosen for all flow simulations presented below. Let us observe that, for this mesh, the classic nondimensional thickness $y^{+}$of the first cell lies between 10 and 100 so that the use of the universal logarithmic wall law is justified. By using this mesh, the time steps, ensuring the calculation convergence, are, respectively, $\Delta t=10^{-5} \mathrm{~s}$ for Cavka and $\Delta t=10^{-4} \mathrm{~s}$ for Fluent.

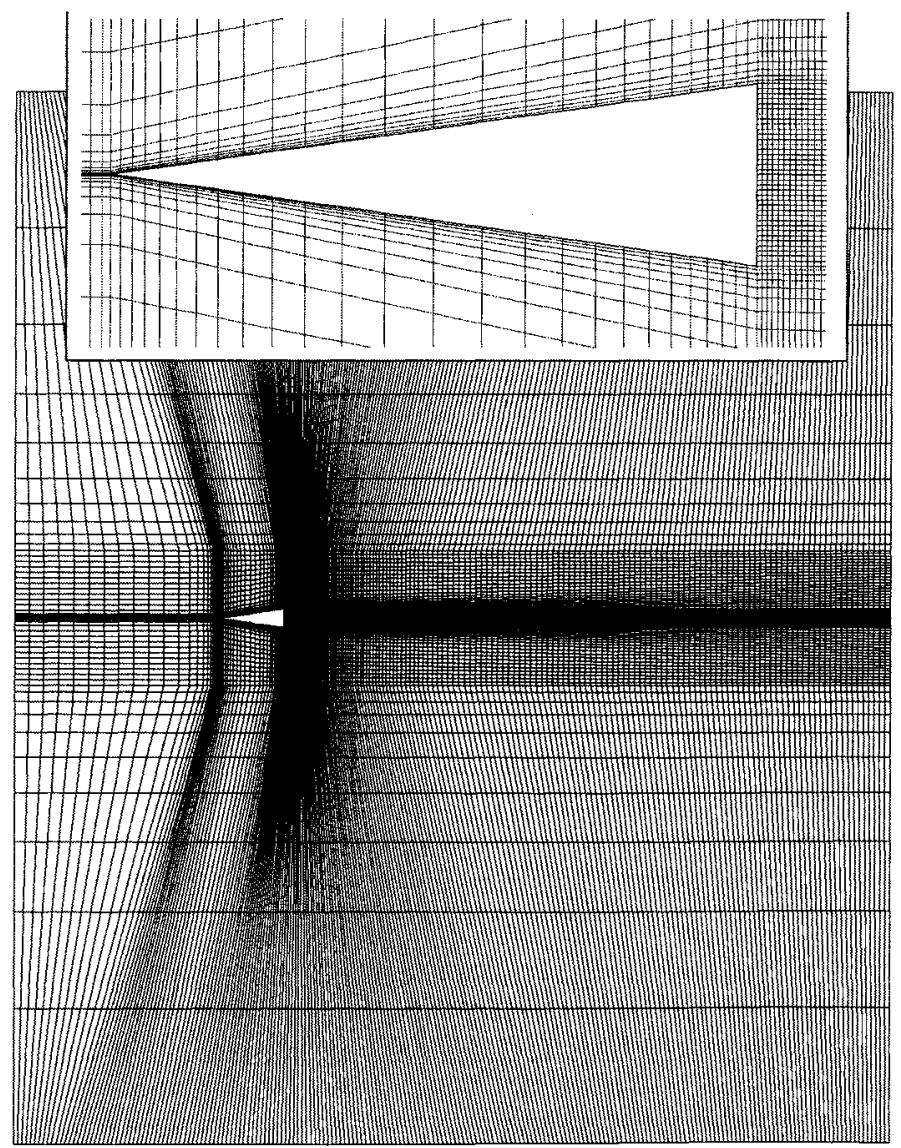

Fig. 2 Computational mesh, $d_{T E}=0.32 \mathrm{~mm}, 18,120$ cells, $H=940 \mathrm{~mm}$ 


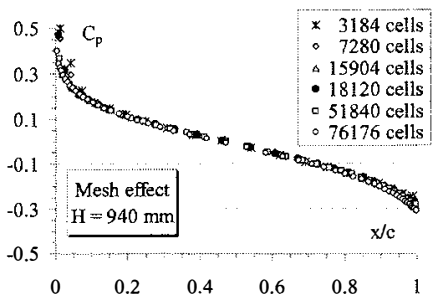

A Pressure coefficient on the wedge

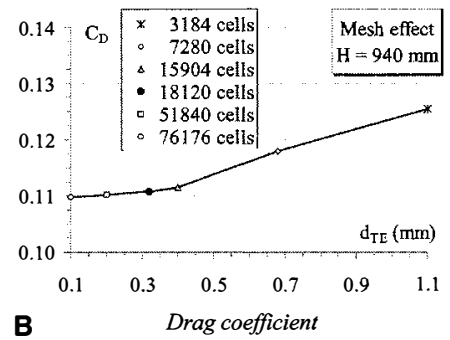

Fig. 3 Mesh effect, steady subcavitating flow, Fluent code, $H=940 \mathrm{~mm}$

This sensitivity analysis with respect to the mesh has been extended to a cavitating case corresponding to $\sigma=0.2$ and $N=10^{8}$ nuclei $/ \mathrm{m}^{3}$. The mesh with 18,120 cells (see Fig. 2) has been refined by dividing their size by a ratio of 2 in both directions. This leads to a new mesh with 72,480 cells that is fine in the region where the cavity is expected. Table 1 shows the influence of the mesh refinement on cavity length, total mass of vapor, and drag coefficient. The relative difference on the mass of vapor is $0.5 \%$, whereas it is about $1 \%$ on cavity length and drag. No noticeable difference was observed either on the void fraction distribution or on the pressure distribution on the foil. Therefore, it can be considered that the mesh with 18,120 cells is fully satisfactory for the cavitating case as well.

\subsection{Influence of the time step}

The sensitivity to the time step has been investigated using Fluent in the case corresponding to a cavitation number of 0.2 and a nuclei density of $10^{8}$ nuclei $/ \mathrm{m}^{3}$. The results are presented in Table 2 . Three values of the time step have been investigated: $10^{-2}, 10^{-3}$, and $10^{-4} \mathrm{~s}$. The cavity closes exactly at the same point of the mesh for all three cases so that no difference is observed on the cavity length. The total mass of vapor in the computational domain decreases slightly when the time step decreases, but becomes stabilized within less than $0.1 \%$ between the two smaller time steps. A similar conclusion is obtained concerning the drag coefficient with an even smaller relative difference of $0.01 \%$ between $10^{-3}$ and $10^{-4} \mathrm{~s}$. This result is not so surprising since the unsteady computation converges toward an almost steady solution that is then independent of time step. The smaller time step has been chosen for all the results presented below.

As a matter of fact, it was observed that both simulations, although fully unsteady, converge toward an almost steady solution. In particular, they do not account for the entrainment of vapor by the liquid flow and the subsequent more or less chaotic shedding of vapor structures that, however, remains confined to the rear part of the cavity and then does not affect significantly the overall cavity shape (Michel 1974). Similar observations were made in the case of self-oscillating cavities that develop at the leading edge of a hydrofoil or in a venturi. Within the framework

Table 1 Influence of mesh refinement on cavity length, total mass of vapor, and drag coefficient $\left(\sigma=0.2, N=10^{8}\right.$ nuclei $\left./ \mathrm{m}^{3}\right)$

$\begin{array}{ccc}\text { Number of cells } & l_{\mathrm{c}} / c & M_{\mathrm{v}}(\mathrm{mg}) \\ 18,120 & 3.1626 & 12.13 \\ 72,480 & 3.1221 & 12.19\end{array}$

72,480

3.1221

12.19

0.08456 0.08354
Table 2 Influence of the time step on cavity length, total mass of vapor, and drag coefficient $\left(\sigma=0.2, N=10^{8}\right.$ nuclei $\left./ \mathrm{m}^{3}\right)$

\begin{tabular}{cccc} 
Time step & $l_{\mathrm{c}} / c$ & $M_{\mathrm{v}}(\mathrm{mg})$ & $C_{\mathrm{D}}$ \\
$10^{-2} \mathrm{~s}$ & 3.1626 & 12.60 & 0.08452 \\
$10^{-3} \mathrm{~s}$ & 3.1626 & 12.14 & 0.08455 \\
$10^{-4} \mathrm{~s}$ & 3.1626 & 12.13 & 0.08456 \\
\hline
\end{tabular}

of a two-dimensional approach, it has been shown that the unsteady behavior of partial cavities strongly depends on the turbulence model. Coutier-Delgosha et al. (2003) have shown that a standard $k-\varepsilon$ RNG model fails at predicting the shedding of cavitation clouds and observed that an artificial reduction of turbulent viscosity in the two-phase regions was necessary to improve the simulation. They also showed that the standard $k$ - $\omega$ model (without compressibility effects) leads to a poor description of the shedding, whereas the inclusion of compressibility in the turbulence modeling leads to a much more realistic behavior. In the present case of supercavitation, it is conjectured that the unsteady behavior of the cavity strongly depends as well on the turbulence model and that the standard $k$ - $\omega$ model used here has a stabilizing effect on the closure region.

Furthermore, some additional aspects of the cavity unsteady behavior could be reproduced only with a three-dimensional model. This is the case for instance of side-jets that develop, together with the traditional counter-current reentrant jet, when the closure line of a partial cavity is not purely two-dimensional even though the general configuration is two-dimensional. As an example, side jets and subsequent secondary three-dimensional (3D) sheddings observed on swept hydrofoils by Foeth (2008) and Foeth and Terwisga (2006a, 2006b) were correctly simulated by Huuva (2008) using an implicit 3D LES technique. It is clear that the present $2 \mathrm{D}$ modeling is unable to simulate the details of the 3D small-scale vapor structures that are formed in the closure region of the supercavity.

\subsection{Effect of confinement}

Since our objective is to simulate an infinite flow field as much as possible, the influence of the tunnel height $H$ is investigated, both under fully wetted conditions and supercavitating conditions.

Figure 4 illustrates, for fully wetted conditions, the effect of tunnel height on pressure distribution and drag coefficient for six different values of $H$ in the range 121 to $1210 \mathrm{~mm}$. Results in Fig. 4 have been obtained using Fluent. From both graphs, it is clear that there is no significant influence of the tunnel height $H$

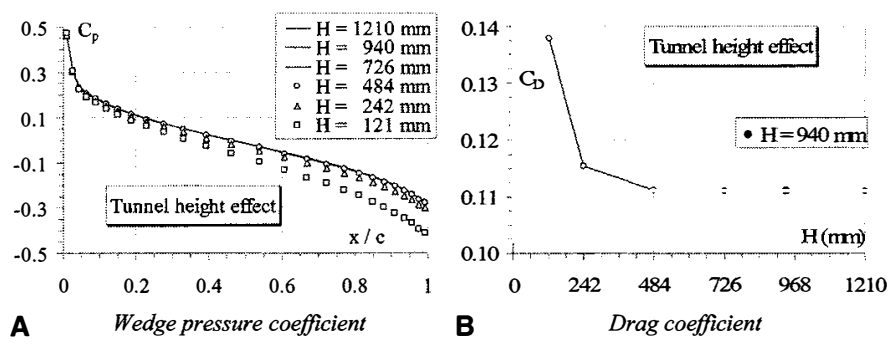

Fig. 4 Tunnel height effect, steady subcavitating flow, Fluent code, $d_{\mathrm{TE}}=0.32 \mathrm{~mm}, 18,120$ cells 

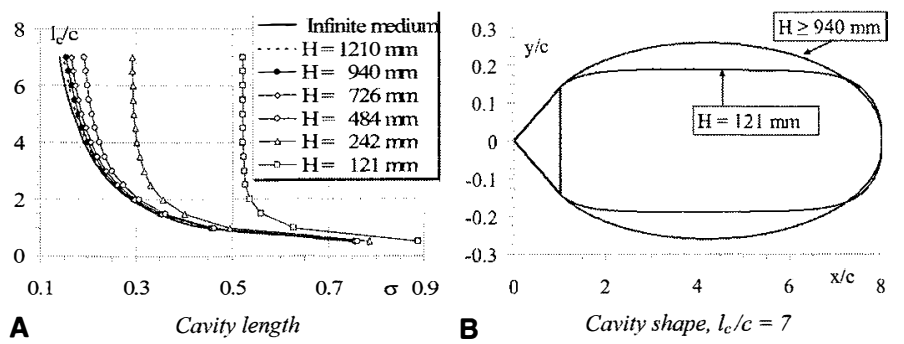

Fig. 5 Tunnel height effect, steady cavitating flow, integral method

above $726 \mathrm{~mm}$. The maximum difference on the pressure coefficient never exceeds $0.1 \%$ for $H>726 \mathrm{~mm}$.

In the supercavitating case, a blockage effect is expected for relatively little values of the ratio $H / c$. As a consequence, the cavity length becomes infinite for a critical nonzero value of the cavitation number. To evaluate this effect, a steady potential flow computation has been developed using an integral method (Pellone et al. 2000). The blockage effect is clearly visible on the computational results presented in Fig. $5 a$. The blockage cavitation number depends on the ratio $\mathrm{H} / \mathrm{c}$. A linearized theory (Cohen et al. 1957) gives the following estimate for the blockage cavitation number in the case of a wedge of vertex angle $\alpha$ (radians):

$$
\frac{\sigma_{\mathrm{b}}}{2+\sigma_{\mathrm{b}}}=\frac{\alpha}{\pi} \cosh ^{-1}\left(e^{\pi c / H}\right)
$$

The values given by equation (15) are in good agreement with results in Fig. $5 a$ and also with experimental results (Michel 1974). As an example, for $H=121 \mathrm{~mm}$, the value given by equation (15) is 0.5 , whereas the numerical prediction of the blockage cavitation number is 0.52 . In addition, Fig. $5 a$ shows that there is a negligible influence of the channel height $H$ on curves $l_{\mathrm{c}}(\sigma)$ for $H \geq 940 \mathrm{~mm}$.

Figure $5 b$ illustrates the influence of the confinement by the tunnel walls on cavity shape. For $H=121 \mathrm{~mm}$, the cavity appears significantly flattened by the relatively close walls, whereas this flattening effect is negligible for $H \geq 940 \mathrm{~mm}$.

As a conclusion, it can be considered that the effect of the tunnel walls are negligible for $H \geq 940 \mathrm{~mm}$. To get results as close as possible to the infinite case, the height of the channel has been chosen equal to $940 \mathrm{~mm}$ for all following computations.

\subsection{Determination of the supercavity boundary}

For RANS computations, the cavity interface is determined from the distribution of void fraction within the flow field. To decide the boundary between liquid and vapor, a threshold has to be chosen for the void fraction. In most cases, the void fraction presents a strong gradient near the cavity interface as shown in Fig. 6. As a consequence, the determination of the cavity boundary is almost insensitive to the chosen threshold as shown in Fig. 7. For all the results presented in section 4, the threshold is chosen equal to $0.5 \%$.

As for the determination of cavity length, several criteria can be used. Figure 8 shows that, in the neighborhood of cavity closure, the drop in void fraction corresponds fairly well to the minimum velocity and to the maximum pressure so that all three criteria can be considered to give almost the same estimate of

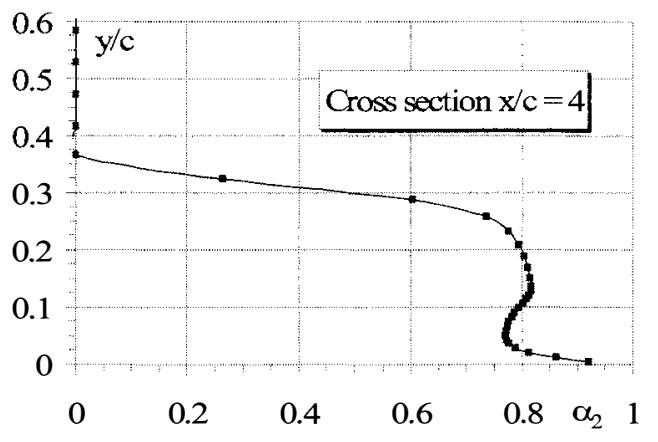

Fig. 6 Void fraction profile, Fluent code, $N=10^{8}$ nuclei $/ \mathrm{m}^{3}, \sigma=0.15$

$\alpha$ void fraction

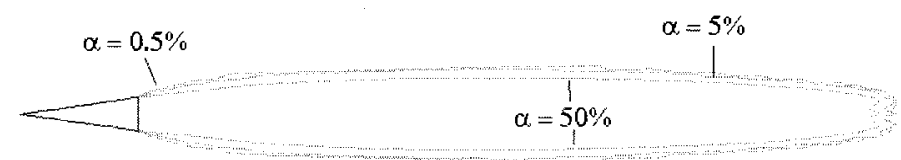

Fig. 7 Influence of the void fraction threshold on cavity shape, Fluent code, $N=10^{8}$ nuclei $/ \mathrm{m}^{3}, \sigma=0.15$

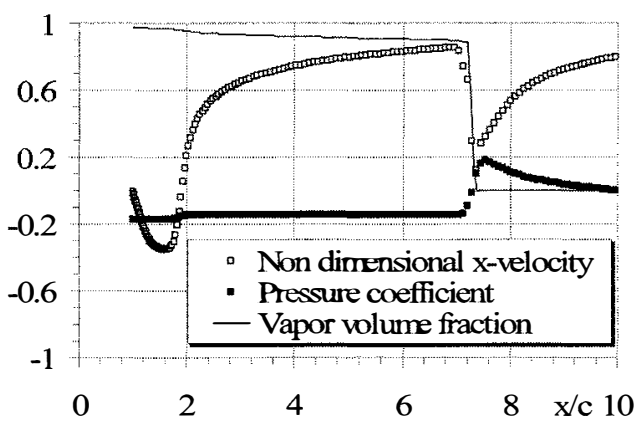

Fig. 8 Cavity length evaluation, three criteria, Fluent code, $N=10^{8}$ nuclei $/ \mathrm{m}^{3}, \sigma=0.15$

cavity length. For all the results presented in section 4 , the cavity closure point is chosen as the first point along the axis of symmetry where the void fraction comes to zero.

Let us observe that, for short cavities (see for instance Fig. 17a), the cavity may present a concave shape around closure. If so, the cavity length measured along the axis of symmetry may slightly underestimate the maximum cavity length that takes place off-axis. The difference is generally small and in any case, the concave shape disappears for long cavities as shown on Fig. 17.

\section{Cavitating results}

\subsection{Cavity length}

Before comparing the cavity lengths obtained by the two RANS codes, it is shown that the cavity length determined by the boundary element method is actually an appropriate reference for discussion. Figure 9 presents a comparison between the curves $l_{\mathrm{c}}(\sigma)$ given by the experiments of Michel (1974), the integral 


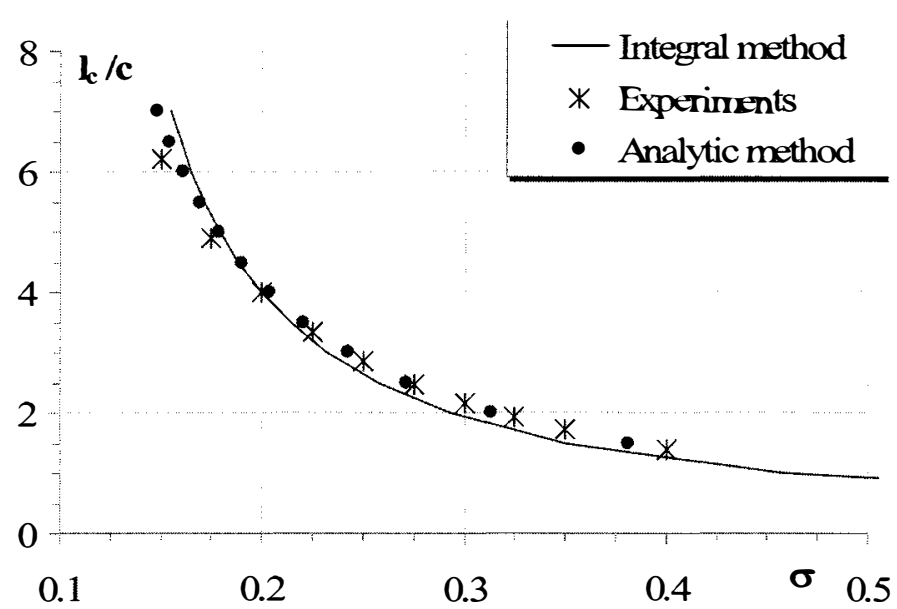

Fig. 9 Cavity length, comparisons

method and an analytic technique. Several analytic techniques have been developed (Tulin 1964) for the computation of supercavity flows. These more or less complicated techniques allow the computation of 2D or axisymmetric, steady or unsteady, supercavity flows. One of the models proposed by Tulin is suitable for a steady supercavitating flow behind a $2 \mathrm{D}$ symmetrical cavitator. Because the model is linearized, the base of the obstacle must be small with respect to its chord, which is the case here. For a wedge of vertex angle $\alpha$ (radians), the cavity length is given by:

$$
\frac{\pi}{\alpha} \frac{\sigma}{2+\sigma}=\sqrt{\frac{c}{l_{\mathrm{c}}}\left(1+\frac{c}{l_{\mathrm{c}}}\right)} \log \left(\sqrt{\frac{c}{l_{\mathrm{c}}}}+\sqrt{\left(1+\frac{c}{l_{\mathrm{c}}}\right)}\right)
$$

Both computational techniques ignore viscous effects that are known to be of minor importance for supercavity flows. It appears that both techniques give very comparable $l_{\mathrm{c}}(\sigma)$ curves, which in addition are very close to the experimental curve. The integral method can however be considered more accurate than the analytic linearized technique because it is nonlinear. It is chosen below for further discussion of RANS results.

RANS computations with Cavka and Fluent have been conducted by decreasing step by step the cavitation number or the downstream pressure $p_{\text {ref. }}$. For each $\sigma$ value, the flow is initialized using the converged previous result obtained at the $\sigma$ value just above. The unsteady computation is started and continued until steady conditions are reached. For the first value of the cavitation number ( $\sigma=0.375$ for Fluent and $\sigma=0.425$ for Cavka), the flow field is initialized using the result of a fully wetted, symmetrical, and steady flow computation. The procedure is illustrated in Fig. 10 where the total mass of vapor within the whole flow field is plotted as a function of time. The successive steps corresponding to decreasing values of the cavitation number are clearly visible. It is also observed that, at the end of each step, the mass of vapor becomes stable and steady conditions are then achieved.

Figure 11 presents a comparison of the evolution of cavity length with cavitation number for both RANS computations. The result of the integral method is also shown for reference. For computations using Fluent and Cavka, the nuclei density was set to $N=10^{8}$ nuclei $/ \mathrm{m}^{3}$. The curves $l_{\mathrm{c}}(\sigma)$ are very comparable between Fluent and Cavka, although a small difference is observed that varies between half a chord length for short cavities

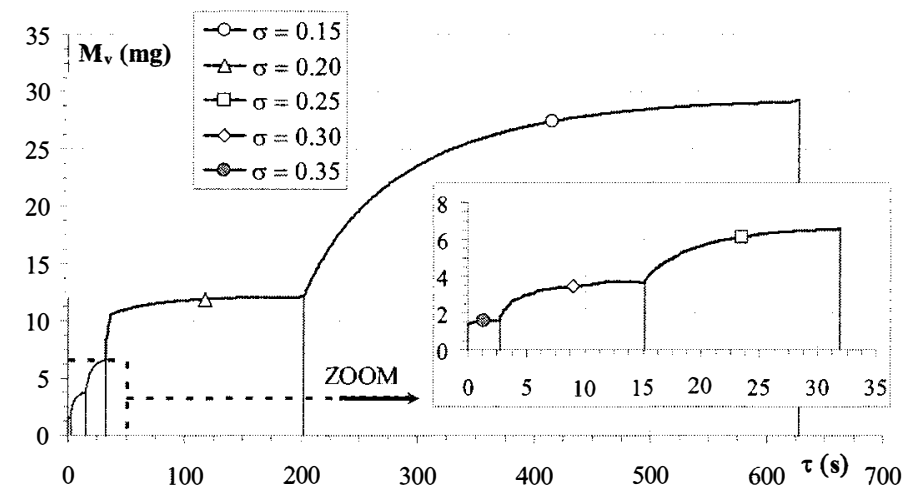

Fig. 10 Vapor mass into the cavity, Fluent code, $N=10^{8}$ nuclei $/ \mathrm{m}^{3}$
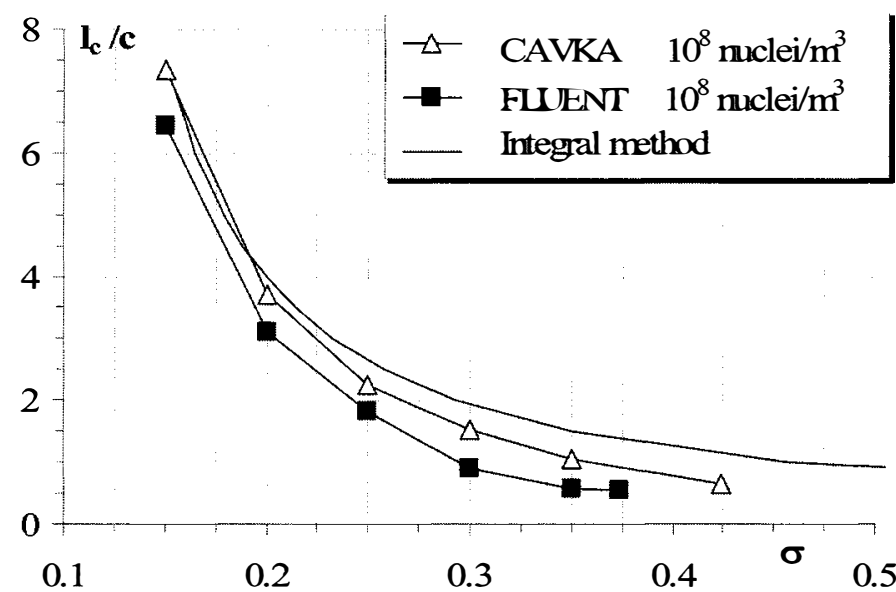

Fig. 11 Cavity length, Fluent and Cavka codes, integral method

$(\sigma>0.2)$ up to about 1 chord length for longer cavities $(\sigma<$ 0.2 ). As shown on Fig. $17 a$, the difference for short cavities is partly the result of the concave shape of the cavity at closure that is more pronounced for Fluent compared with Cavka. Both RANS computations agree fairly well with the integral method. The larger differences are observed for short cavities and are again partly the result of the cavity shape at closure. RANS computations tend to produce a reentrant flow that is responsible for the concave shape of the cavity, whereas this effect is not predicted by the boundary element method.

\subsection{Effect of nuclei density}

As shown by equation (11), the vaporization rate depends on the concentration of nuclei that are supposed to be conveyed by the flow. It can be expected that the production of vapor will decrease with decreasing nuclei density. It appears then important to investigate the effect of nuclei density on the development of cavitation.

Five computations have been conducted with five different values of the nuclei density: $3.10^{5}, 10^{6}, 10^{7}, 5 \times 10^{7}$, and $10^{8}$ nuclei $/ \mathrm{m}^{3}$, all other conditions being unchanged. The evolution of the vapor volume fraction along the horizontal axis of symmetry Ox is plotted in Fig. $12 a$ for $\sigma=0.15$ and the various values of nuclei density considered here. For all cases, a sharp drop in void fraction is observed at cavity closure. On the whole, the void 
fraction inside the cavity slightly decreases with decreasing nuclei density, but remains fairly high, typically between 0.8 and 1 . An increase in nuclei concentration induces an expansion of the supercavity.

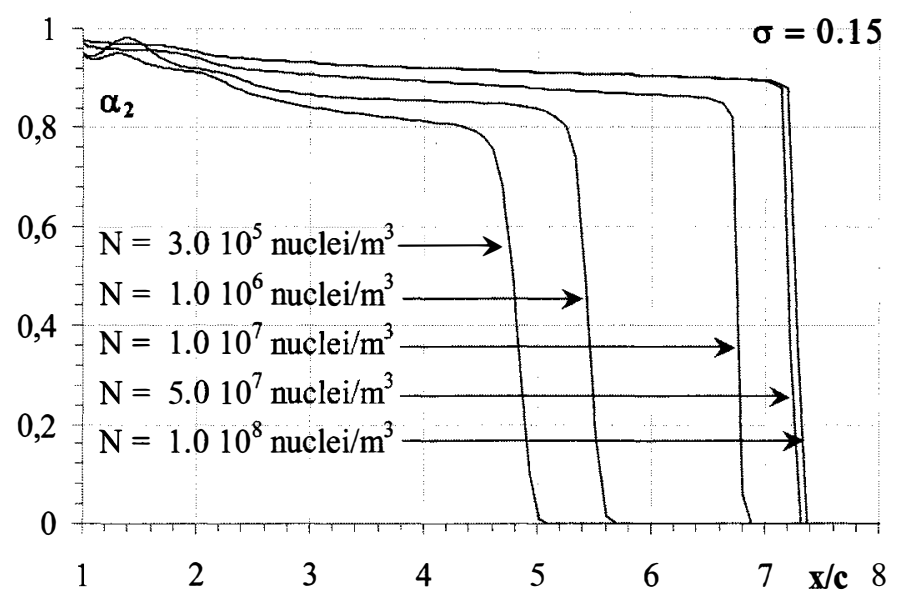

A

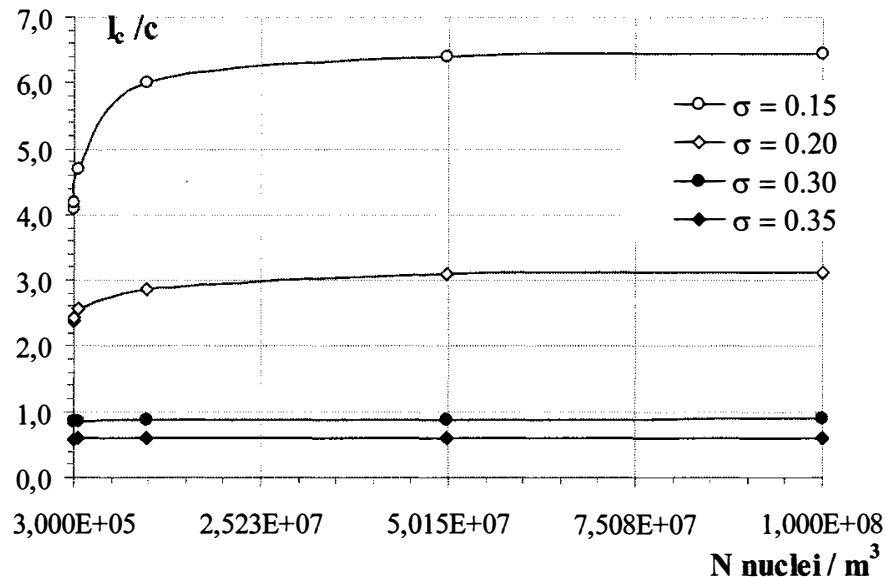

B

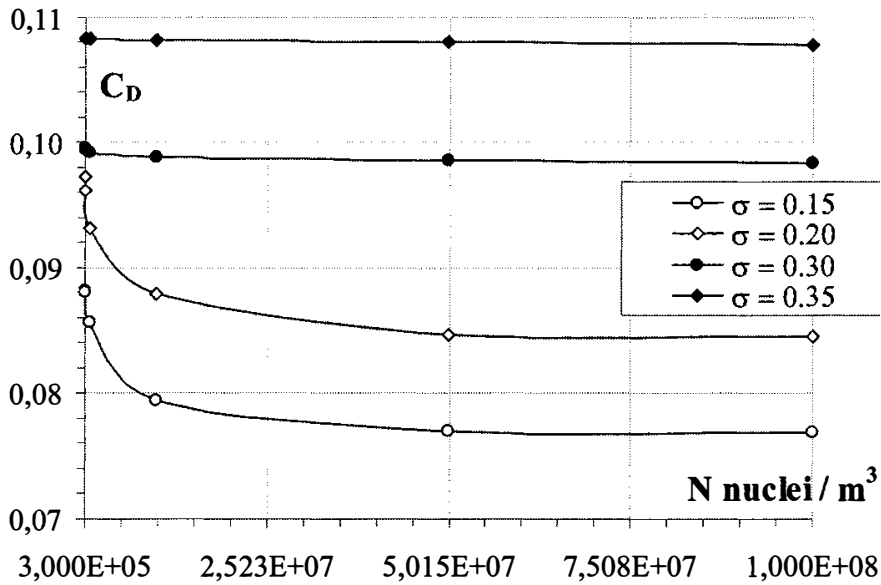

C
This effect is shown in Fig. $12 b$ that represents the variation of cavity length with nuclei density. For the smaller value of the cavitation number $(\sigma=0.15)$, it appears that the cavity length is sensitive to the number of nuclei for small nuclei densities, whereas a saturation effect is observed beyond a critical nuclei density which lies around $10^{8}$ nuclei $/ \mathrm{m}^{3}$. This saturation effect is also visible for larger values of the cavitation number. However, for $\sigma=0.30$ and $\sigma=0.35$; that is, for the two shortest cavities, the asymptotic behavior is already reached for the smallest value of the nuclei density considered here. It is then of practical interest for numerical simulations to consider a sufficiently high value of the nuclei density to limit the influence of this parameter on the results. Figure $12 b$ shows that the critical nuclei density increases with cavity length.

Figure $12 c$ presents the effect of the nuclei density on the drag coefficient. A similar asymptotic behavior to that observed on cavity length is here observed on the drag coefficient. It corroborates the saturation effect obtained for large enough nuclei densities. Thus, the nuclei density has been chosen equal to $10^{8}$ nuclei/ $\mathrm{m}^{3}$ for all the results presented in this section.

\subsection{Drag coefficient}

Drag coefficient is given in Fig. 13 as a function of the cavitation number. For RANS computations, the pressure drag $C_{\mathrm{Dp}}$ and the total drag $C_{\mathrm{D}}$ (including the friction drag) have been plotted. As expected, the friction drag is relatively small compared with the pressure drag. Both RANS computations appear to be in good agreement.

As for the integral method, it gives an evaluation of the pressure drag since the fluid is assumed inviscid. The pressure drag given by RANS computations appears to be slightly greater than that given by the integral method. The difference is mainly the result of the cavity pressure on the obstacle base, which is somewhat smaller than the vapor pressure as confirmed by the Figs. 13 and 14. From an experimental viewpoint, the pressure within a supercavity is almost uniform (except near closure) and equal to the vapor pressure (increased of the partial pressure of noncondensable gas, if any), and this is true also in the vicinity of the wedge base. The difference between the base pressure and the vapor pressure obtained here numerically is actually an artifact of the present computations.

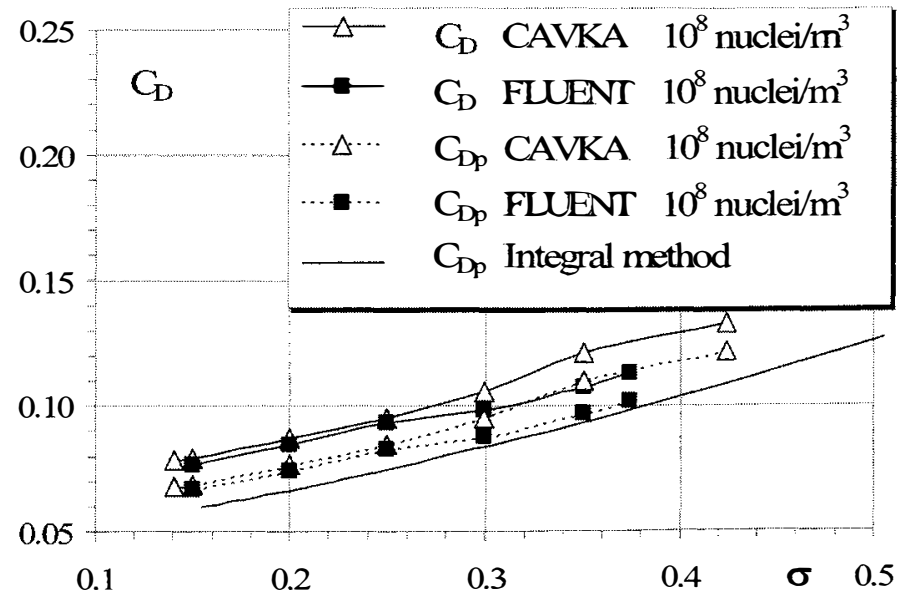

Fig. 13 Drag coefficients: pressure drag and total drag

Fig. 12 Nuclei concentration effect, Fluent code 


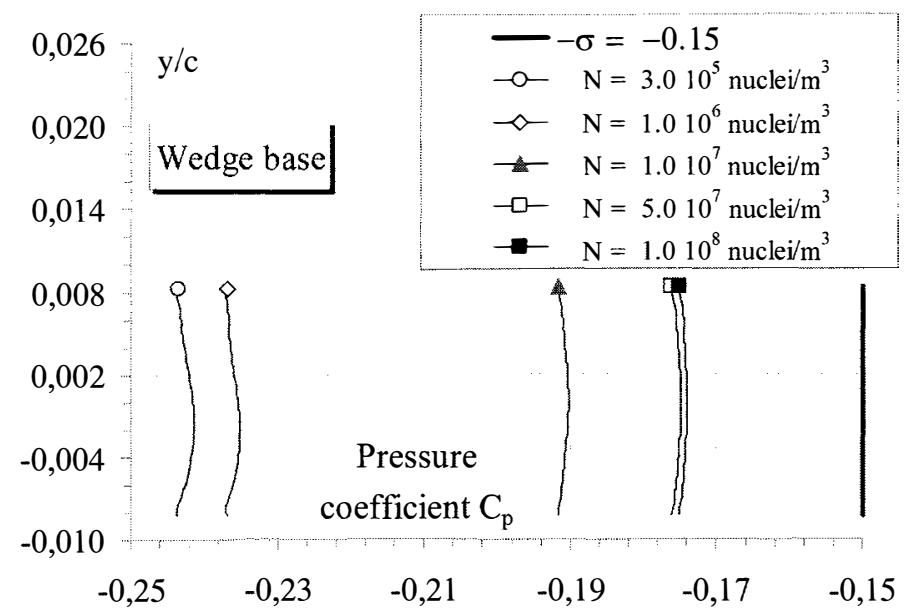

Fig. 14 Wedge base pressure compared with vapor pressure, Fluent code

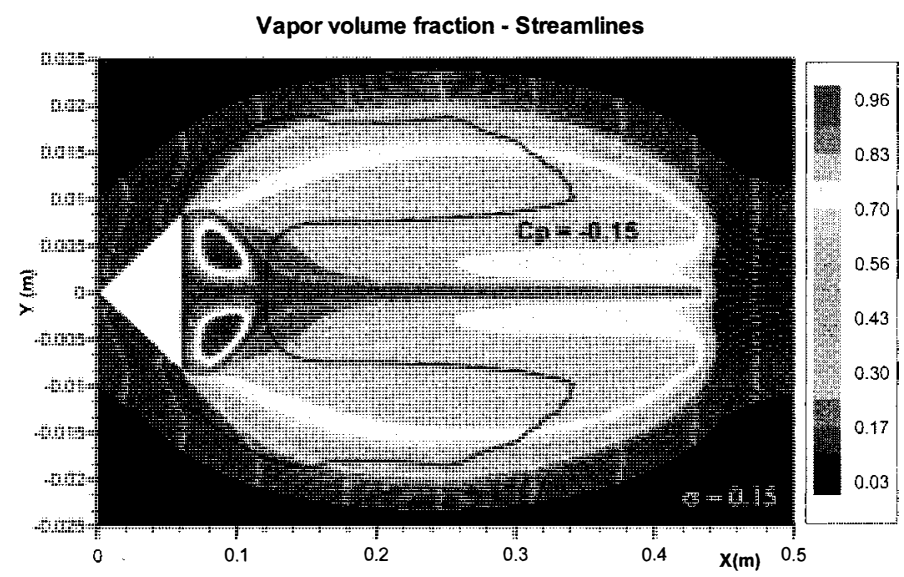

Fig. 15 Streamlines and field of void fraction, $\sigma=0.15$, Fluent code, $N=10^{8}$ nuclei $/ \mathrm{m}^{3}$

In Fig. 15 the black line represents the locus of the points where the pressure equals the vapor pressure (here, $C_{\mathrm{p}}=-\sigma=$ $-0.15)$. Inside the pressure is smaller than the vapor pressure and larger outside. The saturation effect with nuclei density already observed on the cavity length and the drag coefficient is also visible on the pressure at the wedge base in Fig. 14 where the computational results corresponding to various nuclei densities have been plotted.

To further analyze the difference, the pressure drag given by RANS computations has been recalculated by integrating the pressure forces all along the obstacle and assuming that the pressure on the wedge base is exactly equal to the vapor pressure instead of the computed value. Figure 16 shows a clear improvement of the agreement between RANS computations and the integral method.

\subsection{Cavity pressure}

Figure 17 presents the pressure distribution along the axis of symmetry for different values of the cavitation number, together

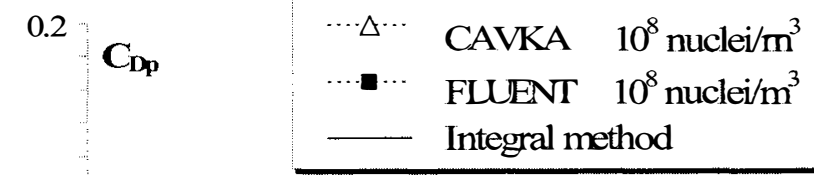

0.15

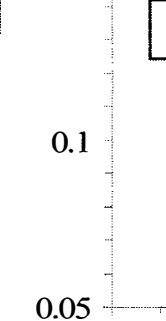

Fig. 16 Pressure drag with $p=p v$ on the wedge back side

with the computed shapes of the supercavity. The cavity pressure is almost constant and equal to the vapor pressure, as expected.

However, a detailed inspection of the evolution of the pressure inside the cavity shows that the pressure just downstream of the obstacle is generally slightly below the vapor pressure. This observation, which was already noted in Figs. 13 and 14 and which is the reason for the small difference between pressure drag coefficients given by RANS methods and the integral method, is quite understandable in the framework of the present bubbly flow model.

According to equation (10), pressure needs to be smaller than the vapor pressure for the bubbles to grow. As shown in Fig. 15, this occurs just downstream of the obstacle and in the vicinity of the cavity interface. Figure 15 shows that streamlines are crossing the domain where $p<p_{\mathrm{v}}$ so that the liquid vaporizes when moving through the interface and feeds the cavity with vapor. In the region where $p>p_{\mathrm{v}}$ and in particular in the closure region where an overpressure is observed, bubbles are collapsing and vapor turns back to liquid.

\subsection{Unsteady behavior}

The unsteady response of the flow to a sudden drop in pressure has been studied using both RANS codes. The starting point is the fully wetted flow, and the cavitation number is supposed to be instantaneously decreased to the value $\sigma=0.2$ and then kept constant until steady conditions are reached. The time evolution of the total amount of vapor in the flow is plotted in Fig. 18. Notice that on this graph the scales in time are different for both curves Fluent and Cavka.

The asymptotic value for a large enough time is comparable for both approaches, but the characteristic rise time is different. It is much longer for Fluent than for Cavka. This shows that the vaporization rate is probably much smaller in the case of Fluent.

As a matter of fact, a vapor mass factor is introduced in Fluent. This factor is a purely numerical parameter that is introduced to avoid divergence of the computation. It is a multiplicative factor in the bubble growth rate equation (10). Its value, which should be unity according to the physics of the phenomenon, must generally be chosen much smaller, typically of the order of 0.001 or even smaller to allow convergence. As a consequence, the explo- 

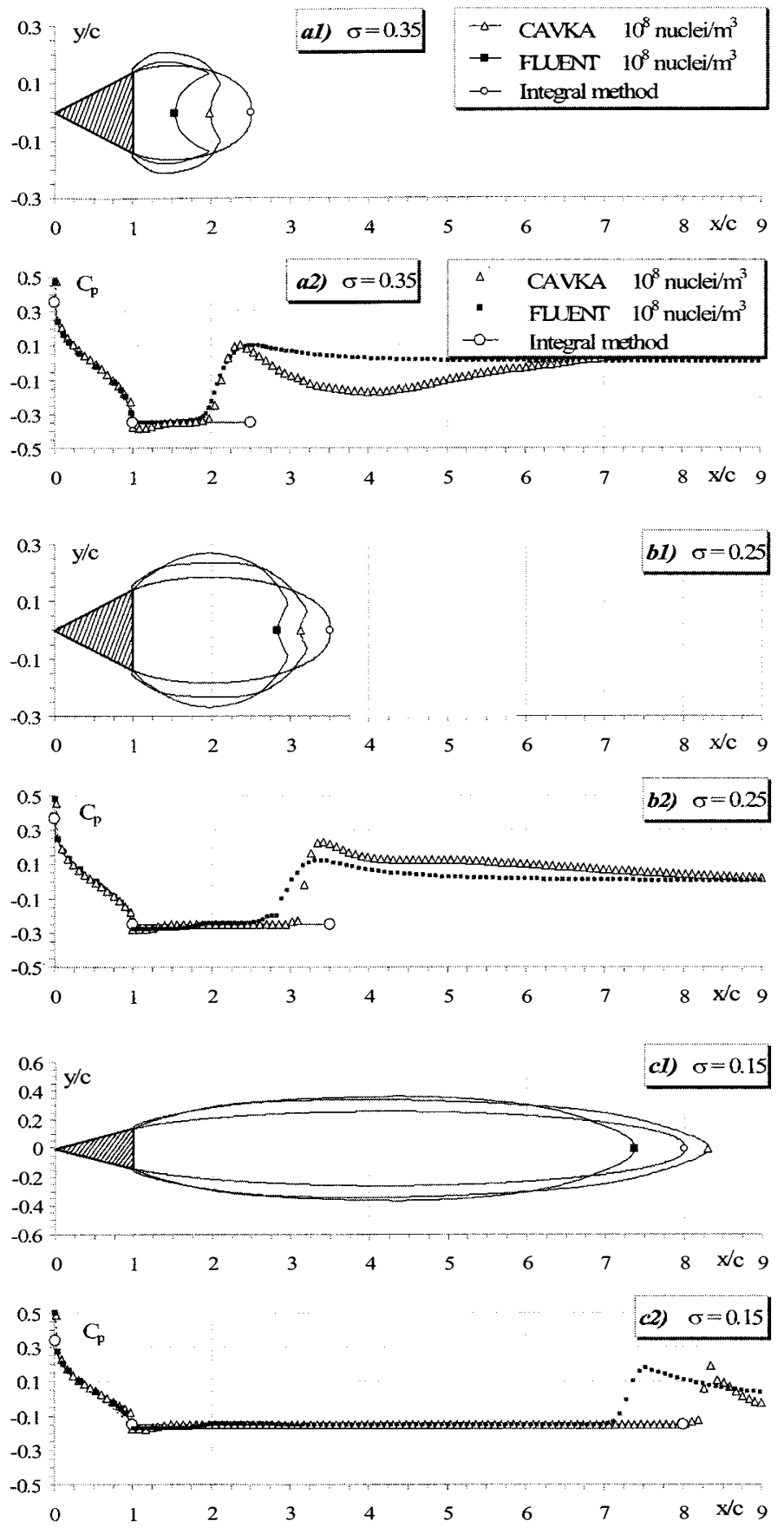

Fig. 17 Cavities shape and pressure coefficient, comparisons

sive growth of cavitation bubbles is damped and the production rate of vapor is underestimated. On the contrary, the numerical scheme used by CAVKA is different and does not require the use of such a damping coefficient. The introduction of this damping coefficient has no consequence on the steady solution but is of the greatest importance for unsteady simulations.

On the basis of the Logvinovich approach (Pellone et al. 2004), it can be inferred that the characteristic response time to a sudden pressure decrease is of the order of the ratio of the cavity length to the flow velocity $l_{\mathrm{c}} / V_{\infty}$. It is the time necessary for the cavity to grow up to its steady length $l_{\mathrm{c}}$. In the present case, the cavity

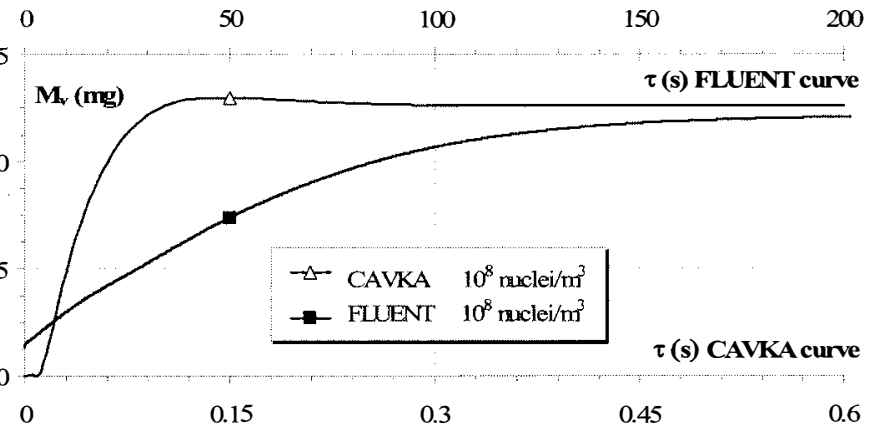

Fig. 18 Evolution of the vapor mass into the cavity, $\sigma=0.2$

length is $l_{\mathrm{c}}=0.22 \mathrm{~m}$ and the flow velocity $V=10 \mathrm{~m} / \mathrm{s}$ so that the characteristic time should be of the order of $0.022 \mathrm{~s}$. The rise time given by Cavka appears to be about 6 times larger (corresponding to $0.13 \mathrm{~s}$ ), whereas that given by Fluent is at least 800 times larger (corresponding to $170 \mathrm{~s}$ ).

\section{Conclusion}

The capacity of conventional RANS techniques to simulate supercavitating flows has been investigated. Two codes have been evaluated, the commercial code Fluent (version 6.0.12) and the code Cavka developed at the Technical University of München. Both codes use the same $k-\omega$ turbulence model and a similar cavitation model based on the asymptotic growth rate of a cavitation bubble. They are applied to the steady and unsteady supercavitating flow around a two-dimensional wedge for which some experimental results are available.

In a preliminary investigation, the influence of mesh refinement has been evaluated under fully wetted conditions. For a number of cells greater than 18,120 , almost no influence of the mesh has been observed on the pressure distribution and on the drag. For further simulations of supercavity flows, both RANS codes have been run using the same mesh of 18,120 cells. The mesh was refined in the wake of the obstacle to track the supercavity more accurately.

A systematic analysis of the influence of the tunnel height has also been conducted under fully wetted and supercavitating conditions to limit the influence of the tunnel walls and simulate as much as possible an infinite flow field. A tunnel height of more than 15 chord lengths has finally been chosen for the simulations.

Since the vaporization rate is a function of the nuclei density, the effect of this parameter was investigated. A saturation effect was observed beyond a critical value of the nuclei density of the order of $10^{8}$ nuclei $/ \mathrm{m}^{3}$, so that it appears preferable to use a high enough value of the nuclei density to reduce the sensitivity of the simulations to this parameter, which is often badly controlled experimentally.

The asymptotic cavity length $l_{\mathrm{c}}$, obtained after a long-term computation and that is then representative of the steady case, was plotted versus the cavitation number $\sigma$. A difference between half a chord length to a maximum of 1 chord length was observed between both simulations, whereas the maximum computed cavity length was about 7 chord lengths. The curves $l_{\mathrm{c}}(\sigma)$ for Fluent and Cavka are in satisfactory agreement with the computational results given by a linearized analytic technique (Tulin 1964) and 
by a nonlinear boundary element method (Pellone et al. 2000) and also with experimental results (Michel 1974). For short cavities, the difference was partly attributed to differences in the shape of the cavity at closure and more especially in its concavity, which results from a reentrant flow.

The total drag given by both RANS simulations agrees within less than $10 \%$. The computed pressure drag is a bit larger than that given by the integral method. The difference is the result of the cavity pressure along the base of the obstacle, which is somewhat smaller than the vapor pressure. This is due to the cavitation model, which requires the pressure in the most upstream part of the cavity to be smaller than the vapor pressure so that the nuclei can grow and actually give birth to a cavity. On the whole, the pressure all along the cavity is, however, almost constant and close to the vapor pressure as expected.

Although steady results are comparable for both codes, the characteristic growth time of the cavity resulting from a sudden drop in pressure from fully wetted conditions is different. The growth time of the supercavity given by Cavka is of the order of $l_{\mathrm{c}} / V_{\infty}$. This is in agreement with the independence principle of cavity expansion derived by Logvinovich in the framework of the slender body approximation. Conversely, the response time of the flow to the same disturbance given by Fluent is several hundreds of times greater. The difference was mainly attributed to the vapor mass factor that appears in Fluent. This is a multiplicative factor that artificially reduces by several orders of magnitude the vaporization rate to prevent divergence of the computation. The result is that the wellknown explosive nature of cavitation is strongly damped and that the growth time of the supercavity is very significantly underestimated. As a conclusion, conventional RANS simulations give satisfactory results for steady supercavity flows. However, a special care has to be taken for the prediction of unsteady supercavitation so that the strong dynamics of cavitation is correctly modeled.

In addition, it was observed that both simulations led to an almost steady solution that does not account for the shedding of vapor structures at cavity closure usually observed experimentally. Turbulence modeling and 3D effects could explain this deficiency. It would be interesting to know whether the introduction of compressibility in the turbulence model would allow a better capture of the unsteady behavior of the supercavity at closure, as it was proved for partial cavities. It would also be interesting to evaluate the capabilities of advanced three-dimensional simulation techniques to predict the detailed structure of the shedding of small-scale vapor structures in the closure region of the supercavity.

\section{Acknowledgments}

The authors are grateful to Professor G.H. Schnerr (Technical University of München) for providing the code Cavka and to E. Sansone for performing the simulations with Cavka.

\section{References}

Birkhoff, G., and Zarantonello, E. H. 1957 Jets, Wakes and Cavities, Academic Press.

Breuer, M., Jovičić, N., AND Mazaev, K. 2003 TranComparison of DES, RANS and LES for the separated flow around a flat plate at high incidence, International Journal for Numerical Methods in Fluids, 41, 357-388.
Briançon-Marjollet, L., Franc, J. P., And Michel, J. M. 1990 Transient bubbles interacting with an attached cavity and the boundary layer, Journal of Fluid Mechanics, 218, 355-376.

Cohen, H., Sutherland, C. D., And Tu, Y. O. 1957 Wall effects in cavitating hydrofoil flow, Journal of SHIP RESEARCH, 1, 3, 31-39.

Coutier-Delgosha, O., Fortes-Patella, R., and Reboud, J. L. 20032003 Evaluation of the turbulence model influence on the numerical simulations of unsteady cavitation, Journal of Fluids Engineering, 125, 38-45.

Coutier-Delgosha, O., Fortes-Patella, R., Reboud, J. L., Hakimi, N., and HiRsCH, C. 2005 Numerical simulation of cavitating flow in 2D and 3D inducer geometries, International Journal for Numerical Methods in Fluids, 48, 135-167.

DiÉVAL, L. 1999 Simulation des écoulements cavitants par poche par une méthode de suivi d'interface (in French), Ph.D. thesis, University of AixMarseille.

Dièval, L., Pellone, C., Franc, J. P., And Arnaud, M. 2000 A tracking method for the modelling of attached cavitation, Comptes Redus de l'Academie des Sciences, 328, IIb, 809-812.

Dular, M., Bachert, R., Stoffel, B., AND ŠIrok, B. 2005 Experimental evaluation of numerical simulation of cavitating flow around hydrofoil, European Journal of Mechanics-B/Fluids, 24, 522-538.

Ferziger, J. H., AND Peric, M. 1996 Computational Methods for Fluid Dynamics, Springer-Verlag, Berlin.

FoETH, E. J. 2008 The Structure of Three-Dimensional Sheet Cavitation, Ph.D. thesis, Delft, The Netherlands.

Foeth, E. J., And Terwisga, T. 2006a The Structure of Unsteady Cavitation, Part I: Observations of an Attached Cavity on a Three-Dimensional Hydrofoil, Ph.D. thesis, Delft, The Netherlands.

Foeth, E. J., AND Terwisga, T. 2006b The Structure of Unsteady Cavitation, Part II: Applying Time-Resolved PIV to Attached Cavitation, Ph.D. thesis, Delft, The Netherlands.

FRANC, J. P., AND Michel, J. M. 2004 Fundamentals of Cavitation, Kluwer Academic Publishers, Dordrecht/Boston/London.

Frikha, S., Coutier-Delgosha, O., ANd Astolfi, J. A. 2008 Numerical investigations of the cavitating flow on two-dimensional hydrofoils: physical modelling methodologies, Proceedings, 12th International Symposium on Transport Phenomena and Dynamics of Rotating Machinery, Honolulu, HI, February 17-22, ISROMAC12-2008-20038.

Greaves, D. 2004 Simulation of interface and free surface flows in a viscous fluid using adapting quadtree grids, International Journal for Numerical Methods in Fluids, 44, 1093-1117.

Huuva, T. 2008 Large Eddy Simulation of Cavitating and Non-cavitating Flow, PhD thesis, Chalmers University of Technology, Sweden.

KInNas, S. A. 2001 Super-cavitating Hydrofoils and Propellers: Prediction of Performance and Design, Von Karman Institute for Fluid Dynamics, RTO AVT/VKI Special Course, Supercavitating Flows, February 12-16.

Kirschner, I. N., Fine, N. E., Uhlman, U. S., Kring, D. C., Rosenthal, B. J., Gieseke, T. A., Kuklinski, R., Varghese, A. N., Stinebring, D. R., Dzielski, J. E., Lindau, J. W., And Kunz, R. F. 2001 Supercavitation research and development, Proceedings, Undersea Defense Technologies, Waikiki, HI, October.

Kubota, A., Kato, H., and Yamaguchi, H. 1992 Modeling of cavitating flows: a numerical study of unsteady cavitation on a hydrofoil section, Journal of Fluid Mechanics, 240, 59-96.

Kunz, R. F., Boger, D. A., Stinebring, D. R., Chyczewski, T. S., And GibeLing, H. J. 1999 Multi-phase CFD analysis of natural and ventilated cavitation about submerged bodies, Proceedings, Third ASME/JSME Joint Fluids Engineering Conference, San Francisco, California.

LEONARD, B. P. 1991 The ULTIMATE conservative difference scheme applied to unsteady one-dimensional advection, Computer Methods in Applied Mechanics and Engineering, 88, 17-74.

Lindau, J. W., Kunz, R. F., Boger, D. A., Stinebring, D. R., and Gibeling, H. J. 2002 High Reynolds number, unsteady, multiphase CFD modelling of cavitating flows, Journal of Fluids Engineering, 124, 607-616.

Merkle, C. L., Feng, J., And Buelow, P. E. O. 1998 Computational modeling of the dynamics of sheet cavitation, in Michel, J. M., and Kato, H., editors, Third International Symposium on Cavitation, Grenoble, France, April 7-10, vol. 2, 307-311.

Michel, J. M. 1974 Etude physique du sillage en écoulement plan, No. 3, La Houille Blanche, 27-43.

Pellone, C., Maître, T., and Briançon-Marjollet, L. 2000 Partially cavitating hydrofoils: experimental and numerical analysis, JouRnal OF SHIP RESEARCH, 44, 1, 40-58. 
Pellone, C., Franc, J. P., And Perrin, M. 2004 Modeling of unsteady 2D cavity flows using the Logvinovich independence principle. CRAS, Mècanique, 332, 827-833.

Pellone, C., AND Rowe, A. 1981 Supercavitating hydrofoils in non-linear theory, Proceedings, Third International Conference on Numerical Ship Hydrodynamics, Bassin d'Essais des Carènes, Paris, France, June.

Persson, T., Bark, G., Bensow, R., Berchiche, N., and Fureby, C. 2006 Large eddy simulation of the cavitating flow around a wing section, Proceedings, Sixth International Symposium on Cavitation, CAV2006, Wageningen, The Netherlands, September.

Pouffary, B., Fortes-Patella, R., And Reboud, J. L. 2003 Numerical simulation of cavitating flow around a 2D hydrofoil: a barotropic approach, Proceedings, Fifth International Symposium on Cavitation, CAV2003, Osaka, Japan, November $1-4$.

Rapposelli, E., AND D'Agostino, L. 2003 A barotropic cavitation model with thermodynamic effects, Proceedings, Fif th International Symposium on Cavitation, CAV2003, Osaka, Japan, November 1-4.

SAITO, Y., NAKAMORI, I., AND IKOHAGI, T. 2003 Numerical analysis of unsteady vaporous cavitating flow around a hydrofoil, Proceedings, Fifth International Symposium on Cavitation, CAV2003, Osaka, Japan, November $1-4$.

SANSONE, E. 2007 Modélisation de la cavitation instationnaire dans les turbines Darrieus (in French), Ph.D. thesis, INP, Grenoble, France.

SAUER, J., AND SchNerR, G. H. 2000 Unsteady cavitating flow-A new cavitation model based on a modified front capturing method and bubble dynamics, Proceedings, 2000 ASME Fluid Eng. Summer Conference, Boston, MA, June 11-15.

Savchenko, Y. N. 2001 Supercavitation-Problems and perspectives, Proceedings, Fourth International Symposium on Cavitation, CAV2001, California Institute of Technology, Pasadena, CA, June 20-23.

Schnerr, G. H., AND SAUER, J. 2001 Physical and numerical modeling of unsteady cavitation dynamics, Proceedings, Fourth International Conference on multiphase flow, New Orleans, LA.

SENOCAK, I., AND SHYY, W. 2002 A pressure-based method for turbulent cavitating flow computations. Journal of Computational Physics, 176, 2 , $363-383$.
SEREBRyakov, V. V. 2002 The models of the supercavitation prediction for high speed motion in water, Proceedings, The International Scientific School "High Speed Hydrodynamics," Cheboksary, Russia, June 16-23, 71-92.

SHI, H., AND Kume, M. 2004 Underwater acoustics and cavitating flow of water entry, Acta Mechanica Sinica, 20, 4, 374-382.

Singhal, A. K., Athavale, M. M., Li, H., And Jiang, Y. 2002 Mathematical basis and validation of the full cavitation model. Journal of Fluids Engineering, 124, 617-624.

Spalart, P. R. 2009 Detached-eddy simulation, Annual Review of Fluid Mechanics, 41, 181-202.

Tulin, M. P. 1964 Supercavitating flows, small perturbation theory, Journal OF SHIP RESEARCH, 7, 3, 16-37.

UbBink, O. 1997 Numerical Prediction of Two Fluid Systems with Sharp Interfaces, Ph.D. Thesis, University of London.

UbBink, O., AND Issa, R. I. 1999 Method for capturing sharp fluid interfaces on arbitrary meshes, Journal of Computational Physics, 153, 26-50.

Vortmann, C., Schnerr, G. H., And Seelecke, S. 2003 Thermodynamic modelling and simulation of cavitating nozzle flow, International Journal of Heat and Fluid Flow, 24, 774-783.

WACLAWCZYK, T., AND KorONOWICZ, T. 2005 Modeling of the flow in systems of immiscible fluids using volume of fluid method with CICSAM scheme, Turbulence, 8, 267-276.

WACLAWCZYK, T., AND KoRONOWICZ, T. 2008 Comparison of CICSAM and HRIC high-resolution schemes for interface capturing, Journal of Theoretical and Applied Mechanics, 46, 2, 325-345.

WANG, G., AND Ostoja-STARZEWSKI, M. 2007 Large eddy simulation of a sheet/cloud cavitation on a NACA0015 hydrofoil, Applied Mathematical Modelling, 31, 3, 417-447.

WILCOX, D. C. 1998 Turbulence Modeling for CFD, DCW Industries, La Cañada, CA.

Wu, J., UtTuRKar, Y., AND SHYY, W. 2003 Assessment of modeling strategies for cavitating flow around a hydrofoil, Proceedings, Fifth International Symposium on Cavitation, CAV2003, Osaka, Japan, November 1-4.

YuAN, W., AND SchnerR, G. H. 2003 Numerical simulation of two-phase flow in injection nozzles: interaction of cavitation and external jet formation, Journal of Fluid Engineering, 125, 963-969. 